\title{
Uma abordagem não destrutiva na fenotipagem de raízes de amendoim
}

Submetido - 25 jul. $2020 \quad$ Aprovado - 07 ago. $2020 \quad$ Publicado - 14 out. 2020
do ${ }_{\text {http://dx.doi.org/10.17648/sas.v1i2.29 }}$

Giovani Greigh de Brito (i)

Engenheiro Agrônomo, Doutor em Fisiologia Molecular de Plantas, Embrapa Algodão/Núcleo Cerrado, Londrina-PR, e-mail: giovani.brito@embrapa.br.

Técnico em Agropecuária, Embrapa Algodão/Núcleo Cerrado, Santo Antônio de Goiás, GO, e-mail: andre.barbieri@embrapa.br.

Engenheiro Agrônomo, Programa de Melhoramento do Amendoim - Embrapa, Santo Antônio de Goiás, GO, e-mail: jair.heuert@embrapa.br.

Taís de Moraes Falleiro Suassuna iD Engenheira Agrônoma, Ph.D em Genética, Programa de Melhoramento do Amendoim - Embrapa, Santo Antônio de Goiás, GO, e-mail: tais.suassuna@embrapa.br.

\section{RESUMO}

Para o amendoim, embora existam esforços que abordam a caracterização da arquitetura e robustez do seu sistema radicular, a maioria destas abordagens estão na dependência de procedimentos destrutivos e altamente demandantes em mão de obra. Neste sentido, nesta primeira etapa do estudo, se propôs demonstrar a praticidade, precisão e possibilidade de caracterização não destrutiva em raízes de amendoim. Foram avaliados o diâmetro médio, área superficial específica, comprimento e volume das raízes de três cultivares e seis linhagens desenvolvidas pelo Programa de Melhoramento do Amendoim da Embrapa, além da cultivar Granoleico. Os resultados obtidos evidenciam que a cultivar BRS $421 \mathrm{OL}$, apresentou o melhor equilíbrio entre, diâmetro médio, comprimento total e volume de raízes, se comparado à média dos demais genótipos analisados (37\% e $200 \%$ superior à média dos demais genótipos avaliados, para volume total e comprimento total de raízes, respectivamente). Evidenciou, nesta cultivar, uma maior densidade de raízes secundárias e terciárias (mais finas) no terço inferior do seu sistema radicular. As cultivares BRS 423 OL e BRS 425 OL e a linhagem 18-2133 OL se destacaram em relação à média e requerem uma caracterização adicional. Devido ao seu desempenho superior para as variáveis morfométricas avaliadas, os genótipos BRS 421 OL, a BRS 425 OL e a linhagem 18-2133 OL já se encontram sendo cultivados sob condições de campo (safra 2019/2020), onde tais variáveis serão mensuradas objetivando confirmar seus desempenhos inicialmente superior.

Palavras-chave: Arachis hypogaea L.; Estresse abiótico; Melhoramento genético.

\section{A non-destructive approach to peanut root phenotyping}

\begin{abstract}
For peanut, although there are efforts that address the characterization of the architecture and robustness of its root system, most of these approaches are dependent on destructive and highly demanding procedures in manpower. In this sense, our purposese was to demonstrate the practicality, precision, and possibility of non-destructive characterization in peanut roots. The root average diameter, root-specific surface area, total root length,and root volume of three cultivars and six advanced lines which were developed by the Embrapa Breeding Program, and the Granoleico genotype were evaluated. The results obtained show that the cultivar BRS 421
\end{abstract}


$O L$ showed the best balance between, root average diameter, total root length and root volume among evaluated genotypes (37\% and $200 \%$ higher than the mean of the others evaluated genotypes, to total root volume and root length, respectively). This cultivar showed a higher density of secondary and tertiary roots (thinner) in the lower third of its root system. The cultivars BRS $423 \mathrm{OL}$, BRS $425 \mathrm{OL}$, and 18-2133 OL line stood out concerning to the average and require additional evaluation. Taking into account their superior performance for the root morphometric evaluated variables, the genotypes BRS $421 O L$, BRS $425 O L$, and 18-2133 OL are already being cultivated under field conditions (2019/2020, growth season), where these variables will be evaluated aiming to confirm their initial performance.

Keywords: Arachis hypogaea L.; Abiotic stress; Genetic breeding.

\section{Un enfoque no destructivo para el fenotipado de la raíz de maní}

\section{RESUMEN}

Para los cacahuetes, aunque hay esfuerzos que abordan la caracterización de la arquitectura y la solidez de su sistema raíz, la mayoría de estos enfoques dependen de procedimientos destructivos y altamente exigentes en mano de obra. En este sentido, en esta primera etapa del estudio, se propuso demostrar la practicidad, precisión y posibilidad de caracterización no destructiva en las raíces de maní. Se evaluó el diámetro promedio, el área de superficie específica, la longitud y el volumen de la raíz de tres cultivares y seis cepas avanzadas desarrolladas por el Programa de Mejoramiento de Embrapa, además del cultivar Granoleico. Los resultados obtenidos muestran que el cultivar BRS 421 OL, presentó el mejor equilibrio entre, diámetro promedio, longitud total y volumen de raíces, en comparación con el promedio de los otros genotipos analizados (37\% y $200 \%$ más que el promedio de los otros genotipos evaluados, para el volumen total y la longitud total de las raíces, respectivamente). Mostró, en este cultivar, una mayor densidad de raíces secundarias y terciarias (más delgadas) en el tercio inferior de su sistema de raíces. Los cultivares BRS 423 OL y BRS 425 OL y el linaje 18-2133 OL se destacaron en relación con el promedio y requieren caracterización adicional. Debido a su rendimiento superior para las variables morfométricas evaluadas, los genotipos BRS 421 OL, BRS 425 OL y el linaje 18-2133 OL ya se están cultivando en condiciones de campo (cosecha 2019/2020), donde estas variables se medirán para confirmar su inicialmente actuaciones superiores.

Palabras clave: Arachis hypogaea L.; Estrés abiótico; Mejora genética.

\section{Introdução}

Mais de $90 \%$ da produção de amendoim (Arachis hypogaea L.) no Brasil utiliza cultivares do tipo Runner (SAMPAIO, 2016). A área atualmente cultivada perpassa por regiões com distribuição irregular de chuvas, englobando desde àquelas onde historicamente se registra ocorrência de veranicos em alguma fase, até outras com acúmulos excedentes de precipitação em uma fase e déficit hídrico em outras. Períodos de estiagens prolongadas tem sido relacionados à retração da produção nos anos de 2014 (SAMPAIO, 2018) e 2019 (SAMPAIO, 2020), bem como dos volumes 
exportados, evidenciando o tamanho do impactos destes fatores climáticos para a cadeia produtiva do amendoim no Brasil.

Impactos da ocorrência do déficit hídrico sobre a produtividade tem sido associado à uma estreita relação com a sua duração e os estágios de desenvolvimento da cultura (REDDY; REDDY; ANBUMOZHI, 2003; WRIGHT; NAGESWARA; FARQUHAR, 1994). Déficit hídrico ocorrendo nas fases terminais do ciclo pode rapidamente esgotar a água disponível ao redor da zona de exploração pelas raízes, impactando sobre o rendimento e a qualidade dos grãos ainda em formação. Estudos evidenciam que enquanto a ocorrência de déficit hídrico nas fases vegetativas causam apenas efeitos menores sobre o crescimento e o rendimento do amendoim (AWAL; IKEDA, 2002), a sua ocorrência em estágios terminais da cultura pode resultar em reduções acima de $20 \%$ na produtividade de grãos (BOONTANG; GIRDTHAI; JOGLOY; AKKASAENG et al., 2010; GIRDTHAl; JOGLOY; VORASOOT; AKKASAENG et al., 2010).

Considerando as funções desempenhadas em processos fundamentais para o desenvolvimento vegetal, esforços para aumentar a compreensão do funcionamento do sistema radicular, além de suas interações edafoclimáticas, e sua plasticidade fenotípica, a qual está sobre controle genético surgem como potencial para contribuir na obtenção de genótipos mais equilibrados para enfrentar as flutuações ambientais, a que são constantemente submetidos ao longo do seu ciclo de cultivo.

Ainda que necessário, as dificuldades para acessar tem pesado para uma ênfase menor em estudos do sistema radicular se comparado ao que está posto para a parte aérea das principais espécies de interesse agrícola (DEN HERDER; VAN ISTERDAEL; BEECKMAN; DE SMET, 2010). Soma-se a isto a escassez de ferramental e técnicas de análise de alta performance, limitando tal abordagem e que necessitam ser superadas (JOSHI; SINGH; HUNT; MACE et al., 2017).

Diante deste cenário, nesta etapa, o estudo objetivou avaliar a aplicabilidade de uma abordagem não destrutiva para a caracterização 
morfométrica em raízes de genótipos de amendoim como uma ferramenta a ser incorporada aos programas de melhoramento genético da cultura.

\section{Material e métodos}

As avaliações não destrutivas do sistema radicular do amendoim foram realizadas em uma estrutura do tipo rizhotrons, instalados em casa de vegetação na sede da Embrapa Soja, em Londrina-PR. Cada rizhotron é constituído por placas de ACM (Alumínio composto) de $3,0 \mathrm{~mm}$ de espessura, composto pela junção de duas placas de alumínio à $0,21 \mathrm{~mm}$ e uma placa de polietileno em seu interior. Esta composição auxilia no isolamento térmico, ao mesmo tempo que confere leveza e resistência ao conjunto.

Cada estrutura Rizhotron possui $60 \mathrm{~cm}$ de altura, $45 \mathrm{~cm}$ de largura e 2,0 cm de espessura (espaço entre uma placa e outra). Em cada rizhotron foi semeado um genótipo. $O$ delineamento experimental empregado foi o inteiramente casualisado, com três repetições. Foram analisados 10 genótipos, dos quais seis linhagens (17-1253 OL, 18-2136 OL, 18-2101 OL, 18-2182 OL, 18-2133 OL e 18-1952 OL) e três cultivares (BRS 421 OL, BRS $423 \mathrm{OL}$ e BRS $425 \mathrm{OL}$ ) foram desenvolvidos pelo programa de melhoramento do amendoim da Embrapa, além da cultivar Granoleico.

Aos 25 dias após a emergência, a irrigação foi suspensa até atingir déficit hídrico moderado, ou seja, quando a taxa de condutância estomática monitorada diariamente, atingiu valores médios ao redor de 200 (mmol m-2 $\mathrm{s}-1$ ), mensurados entre 09-10:00 $\mathrm{h}$ da manhã, valores atingidos aos 13 dias após a imposição do déficit hídrico. A aquisição de imagens ocorreu sob déficit hídrico moderado. Para a aquisição das imagens do sistema radicular, utilizou-se uma câmera fotográfica modelo Canon EOS 7D ISSO 100, Lente $40 \mathrm{~mm}$, posicionados em suporte em L.

Para isto, cada rizotron foi aberto e posicionado frontalmente à lente a uma distância de $1,20 \mathrm{~m}$. As imagens obtidas foram tratadas e posteriormente analisadas por meio do software proprietário WinRhizo PRO 
(WinRhizo Pro 2013, Régent Instruments Inc.). Foram mensurados o volume total de raízes, o seu comprimento total, o diâmetro médio e a sua área superficial específica. Anterior à análise da variância, para cada variável, os dados foram submetidos aos testes de normalidade via Shapiro-Wilk e pelo teste de igualdade da variância. Foram analisados os efeitos de genótipos ( $P$ $<0.05$ ) (One Way Analysis of Variance, via SigmaPlot 14 version) ao final do ciclo de estresse. Os dados estão apresentados graficamente, contendo a média e as barras de seu erro padrão associado.

\section{Resultados e discussão}

Nesta primeira etapa, em que o estudo foi conduzido sob condições de casa de vegetação, foram analisados 10 genótipos, incluindo linhagens do programa de melhoramento do amendoim quanto a variáveis de morfometria e arquitetura radicular em resposta ao déficit hídrico moderado, imposto aos 25 dias após a emergência. Nesta etapa, os resultados obtidos evidenciam que a cultivar BRS $421 \mathrm{OL}$, apresentou o melhor equilíbrio entre, diâmetro médio, comprimento total e volume de raízes, se comparado à média dos demais analisados (Figura 01).

Evidenciou, nesta cultivar, uma maior densidade de raízes secundárias e terciárias (mais finas) no terço inferior do seu sistema radicular, potencialmente conferindo-lhes uma habilidade superior para acessar água em camadas mais profundas do perfil do solo, o que deverá ser avaliado em etapa posterior (Figura 02).

Adicionalmente, as cultivares BRS $423 \mathrm{OL}$ e BRS $425 \mathrm{OL}$ e a linhagem 18-2133 OL se destacaram em relação à média e requerem uma caracterização adicional. Devido ao seu desempenho superior para as variáveis morfométricas avaliadas, os genótipos BRS 421 OL, a BRS $425 \mathrm{OL}$ e a linhagem 18-2133 OL já se encontram sendo cultivados sob condições de campo (safra 2019/2020), para estas mesmas variáveis.

Como já estabelecido, para diferentes espécies vegetais, o sistema radicular do amendoim é integrante importante na aquisição de água e de 
nutrientes do solo, além de comunicar-se com a parte aérea integrando globalmente a estrutura vegetal durante inúmeros processos, inclusive influenciando a dinâmica de trocas gasosas e a fotossíntese. Características constitutivas e/ou induzidas via plasticidade fenotípica podem contribuir para mecanismos importantes, a exemplo o de evitância à seca.

Adicionalmente, em amendoim, a habilidade para explorar camadas mais profundas ao longo do perfil do solo para extrair água tem sido relacionada à maior capacidade para tolerar períodos de escassez de chuvas. Genótipos com sistemas radiculares robustos, capazes de explorar maiores profundidades no perfil do solo e com alta relação raíz/parte aérea tem sido relatados como capazes de manter um maior status hídrico e, como resultante, sustentar maiores rendimentos de grãos quando submetidos ao déficit hídrico (RUCHKER et al., 1995; SOGSRY et al., 2008).

Ressaltaria que por mais robusto que tais resultados possam ser, estes não devem ser extrapolados para todas as situações ambientais pelas quais passam a cultura ao longo de um ciclo de cultivo, já que estes estudos são para condições específicas, além de avaliar um número limitado de genótipos e numa fase também específica de seu desenvolvimento.

Neste sentido, ainda seria importante mencionar que se o caráter robustez do sistema radicular for constitutivo, uma atenção maior deverá ser tomada no sentido de verificar a dinâmica de expressão deste caráter ao longo do seu ciclo. Existem genótipos, em que a coordenação de fonte e dreno; mais especificamente a competição por fotoassimilados entre raízes em crescimento e os órgãos reprodutivos pode resultar na obtenção de plantas altamente tolerantes ao déficit hídrico, contudo, pouco produtivas.

Dessa forma, será importante o desenvolvimento/aprimoramento de ferramental e de protocolos que permitam o monitoramento não destrutivo da dinâmica de desenvolvimento do sistema radicular e sua interação com o solo durante as flutuações no processo de esgotamento e de recarga da água no solo. 
Esta abordagem, por exemplo, poderá permitir também determinar limiares em que seja possível encontrar o melhor equilíbrio nesta dinâmica de desenvolvimento radicular ao longo do ciclo, evitando selecionar plantas que mantenham vigoroso crescimento de raízes durante a fase reprodutiva em detrimento de estruturas reprodutivas, penalizando o rendimento de grãos.
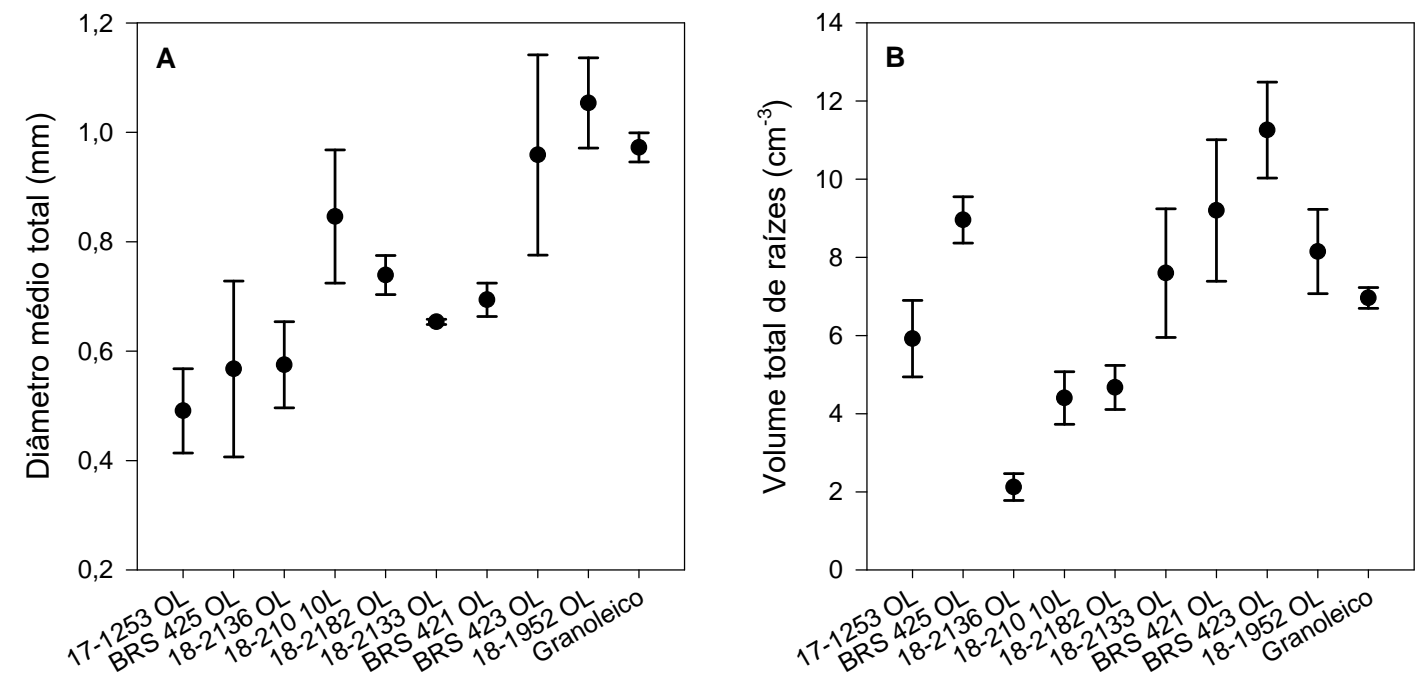

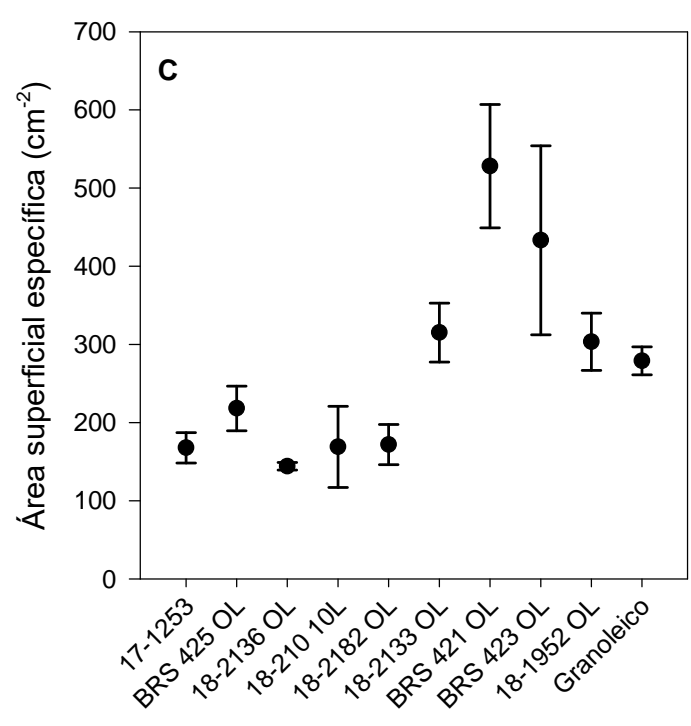

Genótipos

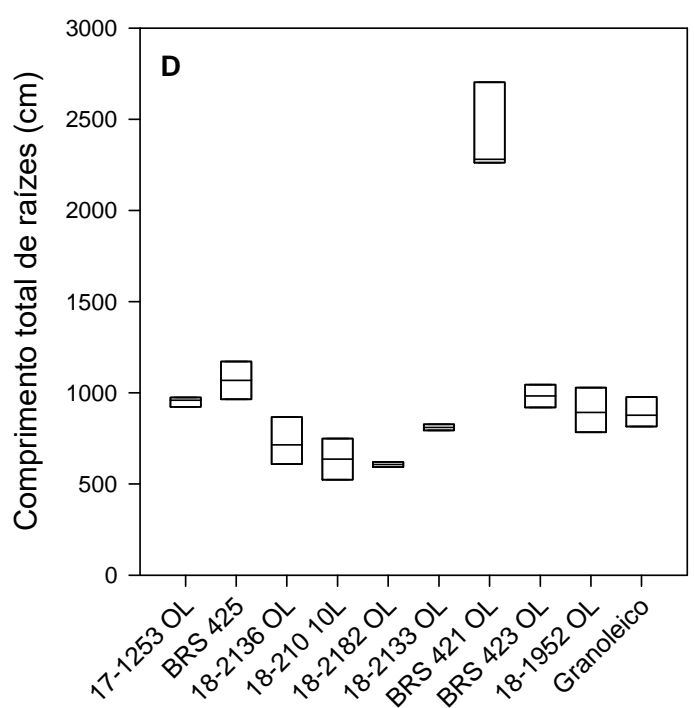

Genótipos

Figura 01. Diâmetro médio (A); volume total de raízes (B); área superficial específica (C) e comprimento total de raízes (D) quantificados em diferentes genótipos de amendoim ao ser submetidos ao déficit hídrico moderado a partir dos 25 DAE. As figuras representam análises efetuadas ao final do ciclo de estresse imposto. As variáveis foram analisadas usando o software estatístico SigmaPlot 14.0. Os dados representam a média de três plantas por tratamento de cada genótipo; as barras de erros indicam o erro padrão da média. 

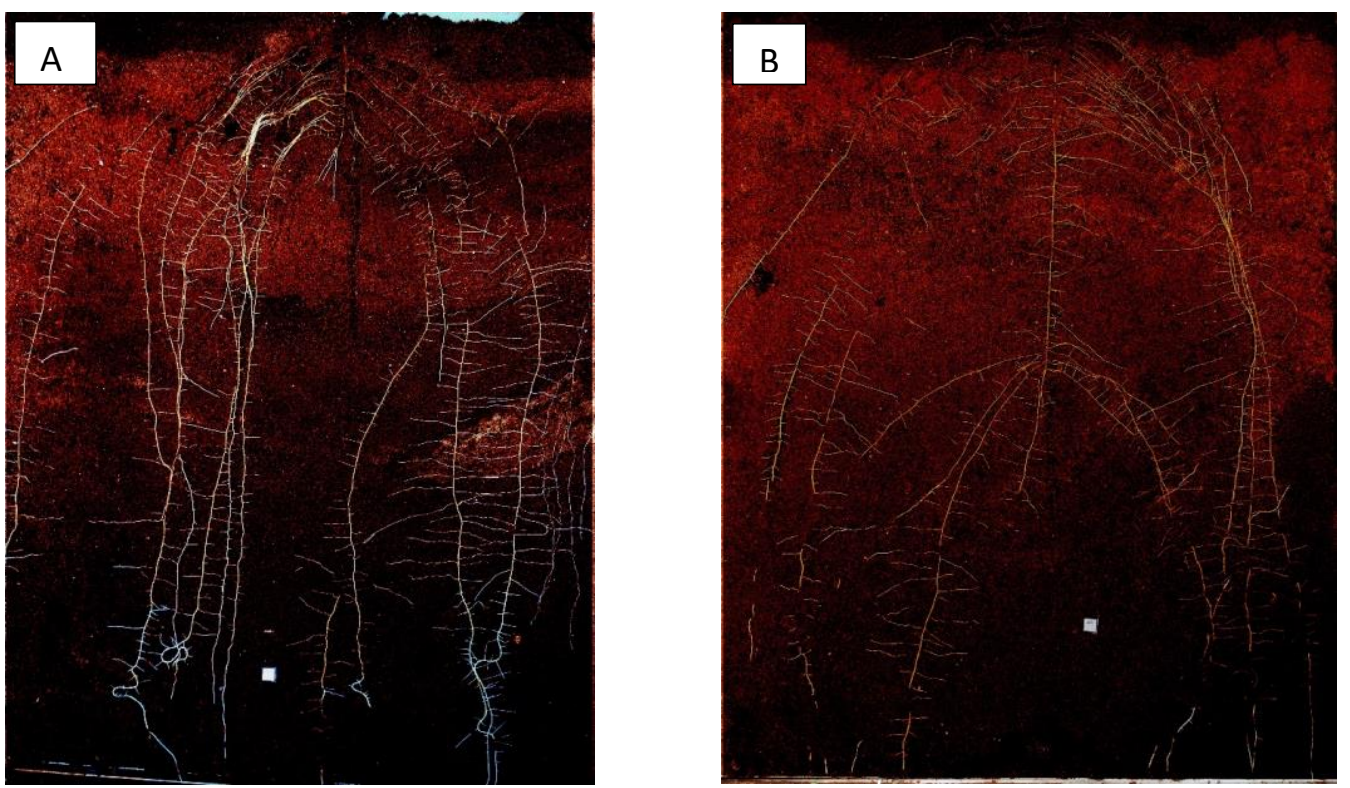

Figura 02. Da esquerda para a direita estão dispostas a Cultivar BRS 421 OL (A) e a linhagem 18-2101 OL (B), ambas imagens adquiridas ao final do ciclo de estresse.

\section{Conclusões}

A abordagem não destrutiva proposta para a fenotipagem em raízes de genótipos de amendoim permite a discriminação da variabilidade existente entre genótipos, mostrando-se adequada às demandas do melhoramento genético. A cultivar BRS $421 \mathrm{OL}$, apresentou o melhor equilíbrio entre, diâmetro médio, comprimento total e volume de raízes, se destacando em relação à média dos demais genótipos analisados.

\section{Agradecimentos}

Este trabalho está vinculado ao Programa de Melhoramento do Amendoim da Embrapa (SEG 20.18.01.021.00). 


\section{Referências}

AWAL, M. A.; IKEDA, T. Recovery strategy following the imposition of episodic soil moisture deficit in stands of peanut (Arachis hypogaea L.). Journal of agronomy and crop science, v. 188, n. 3, p. 185-192, 2002.

BOONTANG, S.; GIRDTHAI, T.; JOGLOY, S.; AKKASAENG, T. et al. Responses of released cultivars of peanut to terminal drought for traits related to drought tolerance. Asian Journal of Plant Sciences, v. 9, n. 7, p. 423-431, 2010.

DEN HERDER, G.; VAN ISTERDAEL, G.; BEECKMAN, T.; DE SMET, I. The roots of a new green revolution. Trends Plant Sci, v. 15, n. 11, p. 600-607, Nov 2010.

GIRDTHAI, T.; JOGLOY, S.; VORASOOT, N.; AKKASAENG, C. et al. Heritability of, and genotypic correlations between, aflatoxin traits and physiological traits for drought tolerance under end of season drought in peanut (Arachis hypogaea L.). Field Crops Research, v. 118, n. 2, p. 169176, 2010.

JOSHI, D. C.; SINGH, V.; HUNT, C.; MACE, E. et al. Development of a phenotyping platform for high throughput screening of nodal root angle in sorghum. Plant Methods, v. 13, n. 1, p. 56, 2017.

REDDY, T. Y.; REDDY, V. R.; ANBUMOZHI, V. Physiological responses of groundnut (Arachis hypogea L.) to drought stress and its amelioration: a critical review. Plant Growth Regulation, v. 41, n. 1, p. 75-88, 2003.

SAMPAIO, R. M. Tecnologia e inovação: evolução e demandas na produção paulista de amendoim. Informações Econômicas, v.46, n. 04, p. $27-42$.

SAMPAIO, R. M. Amendoim: exportações do grão em expansão. Análise e Indicadores do Agronegócio, v. 03, p. 01-05

SAMPAIO, R. M. Amendoim: retração da oferta e queda nas exportações em 2019. Análise e Indicadores do Agronegócio, v.15, n. 02, p. 01-04.

WRIGHT, D. C.; NAGESWARA, R. R.; FARQUHAR, G. D. Water use efficiency and carbon isotope discrimination in peanut under water deficit conditions. Crop Sci., v. 34, n. 1, 1994. 